\title{
SHOCK ORIGIN OF HIGH-VELOCITY MASER EMISSION FROM: CIRCUMNUCLEAR DISKS
}

\author{
EYAL MAOZ AND CHRISTOPHER F. MCKEE \\ Astronomy Department, University of California at Berkeley
}

\begin{abstract}
We propose that the observed high-velocity maser emission from circumnuclear disks arises in spiral shocks, which can naturally account for several peculiar findings in the spectrum and spatial distribution of the maser emission.
\end{abstract}

There is now compelling evidence for masing circumnuclear disks at the centers of NGC 4258 (Miyoshi et al. 1995), NGC 4945 (Greenhill et al. 1997), and NGC 1068 (Greenhill et al. 1996). In all three cases, there is a striking asymmetry in the high-velocity maser spectrum, where the red-shifted high-velocity features are significantly stronger than the blue-shifted ones, therefore suggesting it may be a general phenomenon. We propose (Maoz \& McKee 1998) that the highvelocity maser emission in these systems arises due to shocks in a largely non-inverted disk, which can naturally produce the observed Doppler-shift asymmetry, independent of the existence of a warp in the disk or the azimuthal direction to the observer.

We argue that in circumnuclear disks, the IR line photons which are produced in the maser pump cycle are trapped in the gas due to the large column densities. The re-absorption of these photons drives the level populations into LTE and quenches inversion. The high-velocity masers are suggested to arise in thin masing slabs behind spiral shock fronts in the disk, where the physical conditions are conducive to maser action, and from where the IR line photons can easily escape through the shock front due to the velocity difference between the post-shock and pre-shock gas, thus avoid quenching inversion in that region regardless of the dimensions of the surrounding gas distribution.

Figure 1 illustrates the general geometry, where each spiral region represents a masing slab behind a shock front. Since the maser intensity depends on the path length through an inverted region, the strongest maser emission appears where the l.o.s is tangent to a spiral. The shock fronts must have a trailing geometry due to the action of differential rotation, thus the high-velocity masers are always located in front of the midline in the receding side of the disk, and behind the midline in the approaching side of the disk. Consequently, the blue-shifted beams pass along their path to the observer through a velocity-coherent column of non-inverted gas on the other side of the disk, shown as the shaded region in Figure 1, where absorption occurs. The red-shifted beams never cross such region since the l.o.s velocity of the gas drops monotonically along their path to the observer. This effect is suggested to account for the observed Doppler-shift asymmetry. The model predicts a continuous, outward drift in the positions of all the high-velocity maser features due to the slow rotation of the spiral waves. In NGC 4258 , where we estimate a typical pitch angle of about $2.5^{\circ}$, the predicted rate is $\approx 0.6 \mu \mathrm{as} \mathrm{yr}^{-1}$, which should result also in a systematic decline in velocity at a rate of $\approx 0.05 \mathrm{~km} \mathrm{~s}^{-1} \mathrm{yr}^{-1}$. If the positions of the high-velocity masers in the disk can be accurately determined, we expect the red-shifted ones to appear slightly in front of the midline, and the blue-shifted ones slightly behind it. The model also implies that the high-velocity maser spots should appear clustered in distinct clumps rather than being uniformly or randomly distributed. The clumps may either be narrow or broad, depending on whether the the shock fronts are heavily corrugated and thereby effectively thickened. The estimated mass accretion rate due to energy dissipation in the shocks is consistent with that of the advection-dominated accretion flow suggested for NGC 4258. The model introduces only negligible corrections to the previously derived black hole mass and galaxy distance. 


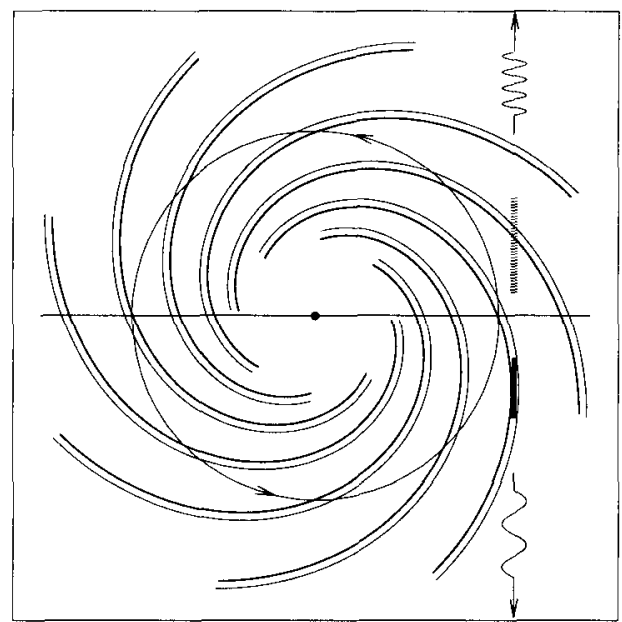

Figure 1. Illustration of the general geometry (see text).

The low-velocity maser emission in NGC 4258 is suggested to arise in a ring of masing gas at the inner edge of the disk, where the direct X-ray irradiation by the central source may provide the energy that ultimately powers the masers. This may explain why all the low-velocity maser sources are confined to a narrow annulus at the inner edge of the disk, although velocity coherence is maintained along the entire length of the minor axis of the projected disk.

\section{References}

Greenhill, L. J., et al. (1996) VLBI Imaging Of Water Maser Emission From The Nuclear Torus Of NGC1068, ApJ, Vol. no. 472, pp. L21-L24.

Greenhill, L. J., Moran, J.M. \& Herrnstein, J. R. (1999) The Distribution Of Water Maser Emission In The Nucleus Of NGC $4945, A p J$, Vol. no. 481, pp. L23-26.

Maoz, E. \&-McKee, C. F. (1998) Doppler-Shift Asymmetry In High-Velocity Maser Emission From Shocks In Circumnuclear Disks, To appear in ApJ, February 10th, 1998

Miyoshi, M., et al. (1995) Evidence For A Black Hole From High Rotation Velocities In A Sub-Parsec Region Of NGC4258, Nature, Vol. no. 373, pp. 127-129 